\title{
Spinodal of supercooled polarizable water
}

\author{
P. Gallo ${ }^{\dagger *}$, M. Minozzi ${ }^{\dagger \dagger}$ and M. Rovere ${ }^{\dagger}$ \\ $\dagger$ Dipartimento di Fisica, Università "Roma Tre", \\ and Democritos National Simulation Center, \\ Via della Vasca Navale 84, 00146 Roma, Italy \\ $\ddagger$ CNR Istituto di Acustica "O. M. Corbino", \\ Via del Fosso del Cavaliere 100, 00133 Roma, Italy
}

\begin{abstract}
We develop a series of molecular dynamics computer simulations of liquid water, performed with a polarizable potential model, to calculate the spinodal line and the curve of maximum density inside the metastable supercooled region. After analysing the structural properties, the liquid spinodal line is followed down to $T=210 \mathrm{~K}$. A monotonic decrease is found in the explored region. The curve of maximum density bends on approaching the spinodal line. These results, in agreement with similar studies on non polarizable models of water, are consistent with the existence of a second critical point for water.
\end{abstract}

PACS numbers: 61.20.Ja,64.70.My,64.70.Fx

\section{INTRODUCTION}

Liquid water behaviour as a function of temperature and pressure differs from that of most liquids. As a major example the presence of a line of maximum density (TMD line) in the $\mathrm{P}-\mathrm{T}$ phase diagram is most significant, as it divides the entire $\mathrm{P}-\mathrm{T}$ phase diagram into two regions with remarkably different properties: the coefficient of thermal expansion is negative on the low-temperature side of the TMD line, while it is positive on the high-temperature side. Similarly, several other thermodynamic and dynamical quantities show peculiar properties. For instance the isothermal compressibility and heat capacity exhibit a minimum as a function of temperature and show an anomalous behavior more pronounced in the supercooled liquid state [1]. When decreasing temperature in fact the coefficient of thermal expansion, the isothermal compressibility and the constant-pressure specific heat increase rapidly. These quantities appear to diverge at a temperature $T \approx-45 C$ if they are extrapolated below the lowest temperatures at which they are measurable $(T=-42 \mathrm{C}$ at $P=1$ bar $)[1]$. The supercooled region below $T=-42 \mathrm{C}$ is experimentally unreachable due to the strong tendency of water to crystallize. It is nonetheless believed that nucleation in supercooled water might be due to the presence of impurities that drive the liquid toward the more stable phase [2]. Therefore the experimentally unreachable zone of supercooling is believed to be physically significant. Measurements of the rate of evaporation on amorphous water have in fact proved that the amorph can be connected with normal liquid water by a reversible thermodynamic path at atmospheric pressure [3]. Besides at ambient pressure experiments have proven the existence of supercooled liquid water close to the glass transition temperature [4].

*Author to whom correspondence should be addressed; e-mail: gallop@fis.uniroma3.it
In spite of all the interest driven by these anomalies a coherent theory of the thermodynamic and transport properties of supercooled water does not yet exist, also due to the difficulties encountered in experiments.

Different thermodynamic scenarios have been proposed through the years for the peculiar metastable behaviour of water [5]. Among these three of them have received a great attention in literature. The stability limit conjecture (SLC) is the first scenario proposed. It attributes the anomalies of water to the presence of a continuous retracing spinodal curve, bounding the superheated and supercooled states. The spinodal, which represents the limit of mechanical stability of a liquid, is hypothized to retrace to higher pressure values below a temperature at which it intersects the locus of the TMD [6, 7]. The second critical point scenario (SCP), based on extrapolation of simulated data, ascribes the anomalous properties of water to the presence of a metastable, low temperature liquid-liquid critical point, associated with a phase transition between a low-density and a high density liquid phase [8]. Experimental evidences of the existence of two liquid phases compatible with a second critical point have been presented [9]. The singularity free scenario (SF) explains the behaviour of supercooled water with the presence of anomalous fluctuations due to the hydrogen bonds and no underlying singularity is invoked [10]. The SF assumes that upon isobaric cooling the thermodynamic response functions go through a maximum but remain finite. An anomalous increase in isothermal compressibility, heat capacity, and thermal expansion is explained by the existence of the density maxima locus, which is negatively sloped in the $P, T$ plane [2]. According to this scenario, no phase transition or critical point occurs at low temperatures.

A fundamental role in the clarification of the scenario of supercooled water is played by the study of the behaviour of the spinodal line, and its relation with the line of TMD. Due to the limitations of the experiments in the supercooled realm, in order to assess the different hypothesis numerical studies have become of uttermost im- 
portance. Many computer simulation studies indeed have been performed with several different water site models, ST2,TIP4P,SPC/E and TIP5P [11, 12, 13, 14, 15, 16]. All the above models do not take into account explicitly the polarizability of the water molecules. Polarizable models for water have been also developed [17, 18, 19, $20,21,22,23,24,25,26,27,28,29$ ] to give a more realistic description of the behaviour of the system in different thermodynamic conditions. Depending on the zone of the phase diagram investigated they have proven to be equally or more realistic to describe the features of water. Studies of the behaviour of the spinodal line for polarizable water model potential have been never carried out. It is therefore of interest to study the behaviour of the spinodal line for potentials where polarizability is explicitly taken into account.

We present here a Molecular Dynamics, MD, study of the spinodal and the TMD line of supercooled water performed with the polarizable potential, introduced in the literature by Ruocco and Sampoli 22] and parametrized by Brodholt, Sampoli and Vallauri [23], the BSV potential. We found that the BSV model is appropriate for our study since it reproduces the thermodynamical properties of water in the region of the gas-liquid coexistence better than other polarizable models over a broad temperature range 30]. It has also been shown that within the BSV model the site-site radial distribution functions are in good agreement with the experimental data in a broader range of thermodynamical conditions. [31, 32, 33, 34, 35]

In the next section we describe the computer simulation in details. After presenting in the third section the structural properties of the system upon supercooling, the behaviour of the spinodal and of the TMD lines are reported in the fourth section. Last section is devoted to conclusions.

\section{COMPUTER SIMULATIONS}

We performed MD computer simulations of water with the Model 4 of ref. [23]. An induced polarizable dipole moment $P$, located in the center-of-mass of the molecule, describes the effect of the electric field of the environment on the molecules. The induced dipole $p_{i}=\alpha E_{i}$ is calculated from the local electric field $E_{i}$ with an iterative procedure by assuming an isotropic polarizability. The value of this polarizability is fixed to the single molecule value $\alpha=1.44 \AA^{3}[22]$.

Each simulation is conducted in the $N V T$ ensemble with 256 water molecules enclosed in a cubic box with periodic boundary conditions. The simulations have been performed with the minimum image convention and a cut-off of the interactions at half of the box length. The long range part of the electrostatic interactions is taken into account with the reaction field method. Details of the extension of the reaction field method to include polarization effects can be found in references [22, 36]. The time step $\delta t$ for the integration of the molecular trajectories is fixed at $1 \mathrm{fs}$.

To analyse the phase diagram and the spinodal curve inside the metastable supercooled region we carried out isothermal simulations for eight different temperatures: 350, 300, 280, 260, 240, 230, 220 and 210 K. For each eight different densities are simulated, namely 1.05, 1.00, $0.98,0.95,0.90,0.87,0.85$ and $0.83 \mathrm{gr} / \mathrm{cm}^{3}$. The box length $L$ spans from $L=19.39 \AA$ to $L=20.96 \AA$ to cover the range of investigated densities.

During equilibration the temperature is controlled via a velocity rescaling procedure. Production runs are performed in the microcanonical ensemble. For the temperatures and the densities closer to the spinodal line the longest equilibration time required was of $5 \mathrm{~ns}$.

In tables $\square$ and 1 we report the temperatures, densities, pressures and internal energies of the simulations we have performed.

\section{STRUCTURAL PROPERTIES}

Structural quantities have been calculated averaging from a minimum of $2.5 \times 10^{3}$ configurations at $T=300 \mathrm{~K}$ to a maximum of $3 \times 10^{3}$ configurations at $T=220 \mathrm{~K}$, all equally spaced and taken every $2 p s$. We have calculated the site-site radial distribution functions (RDF) $g_{\alpha \beta}(r)$ to monitor the internal structure at every temperature. We report $g_{O O}(r), g_{O H}(r)$ and $g_{H H}(r)$ for $T=300 \mathrm{~K}$ in Fig 1 and $g_{O O}(r)$ for $T=220 \mathrm{~K}$ in Fig 2 calculated at several densities. At $T=300 \mathrm{~K}$ the peak positions agree with the experimentally measured structure of water [37]. Upon decreasing temperature the peaks remain at the same position and become sharper. By varying

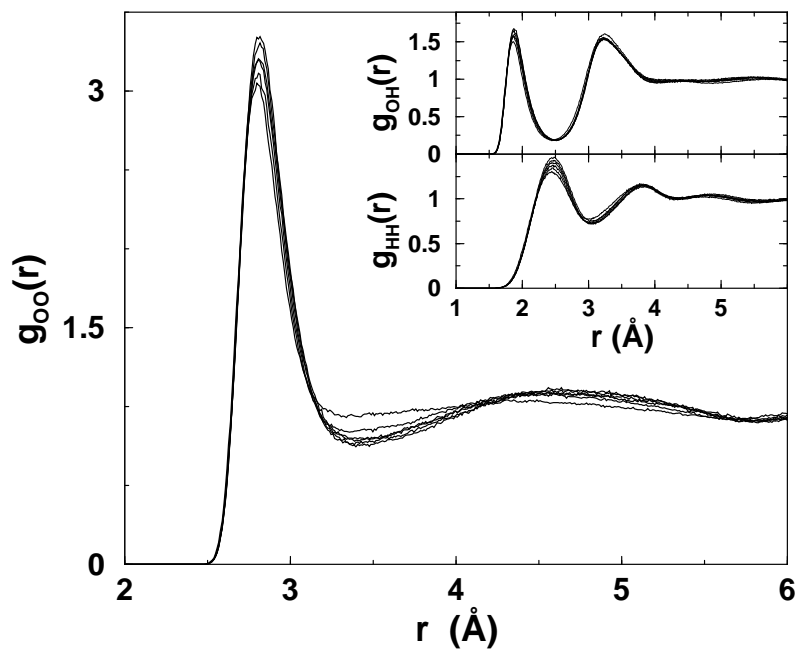

FIG. 1: Site-site pair correlation functions of the BSV water at $T=300 \mathrm{~K}$ and densities $\rho=1.00,0.98,0.95,0.90,0.87$, $0.85,0.83 . g_{O O}(r)$ is in the main frame. The insets show $g_{O H}(r)$ and $g_{H H}(r)$ starting from the top.

the densities we do not observe very large changes in the 
$g_{\alpha \beta}(r)$ apart for an increase in the height of the peaks, even when the system approaches the spinodal line (see next section). This is an important test to verify the absence of disomogeneities or cavitation phenomena close to the limit of mechanical stability. A further check has been performed with the calculations of the local density fluctuations. For this sake we have divided our simulation box into 64 sub-boxes and calculated the average distribution functions of the local density (not shown). In all cases we do not find deviations from a gaussian distribution centered on the fixed density of the system.

A deeper insight in the local ordering of the atoms can be achieved by calculating the coordination numbers. In the upper inset of Fig. 2 we show $n_{O O}$, the coordination number of the oxygens, as a function of $\rho$ :

$$
n_{O O}=4 \pi \rho \int_{0}^{r_{\min }} g_{O O}(r) r^{2} d r
$$

where $r_{\min }$ is the value of the interatomic distance at which the first minimum in $g_{O O}(r)$ is located. It is important to observe the different behaviour of $n_{O O}$ at the two temperatures $T=300 \mathrm{~K}$ and $T=220 \mathrm{~K}$ on lowering the density. At $T=300 \mathrm{~K}$ for the highest density $n_{O O}$ results to be 5.2. This value is the signature of an open network of water, where also interstitial molecules are present 33]. $n_{O O}$ then decreases until it reaches the limiting value 4 characteristic of the local tetrahedral order. At the lower temperature $T=220 \mathrm{~K} n_{O O}$ is found to be quite constant around the value 4 . These findings indicate that the open network of $H$-bonds becomes better defined as $\rho$ and/or $T$ decrease.

In connection with the tendency of the $H$-bond network to become more ordered it is expected a sharpening of peaks of the RDF. A quantitative measure of this sharpening can be obtained looking at the value of the RDF at its first minimum, $g\left(r_{\min }\right)$. As a consequence we also expect to observe a decrease of $g\left(r_{\min }\right)$ as the coordination shells become better defined. In the lower inset of Fig. 2 we observe instead the presence of a minimum in the behaviour of $g\left(r_{\min }\right)$ as function of the density. The minimum is more evident at $T=220 \mathrm{~K}$. This effect evidences an important change in the thermodynamical properties of the system, since it is related to the behaviour of the entropy in the vicinity of the spinodal, as previously discussed in literature [11]. Therefore we can consider that the change of slope in the $g\left(r_{\text {min }}\right)$ curve is a signature of the approaching of the system to the limit of mechanical stability.

\section{SPINODAL LINE}

The limit of the mechanical stability of a system can be determined from a study of the isothermal compressibility. In the space of variables identifying the thermodynamic state of the system in fact, the condition for the mechanical stability is [2]

$$
K_{T}>0
$$

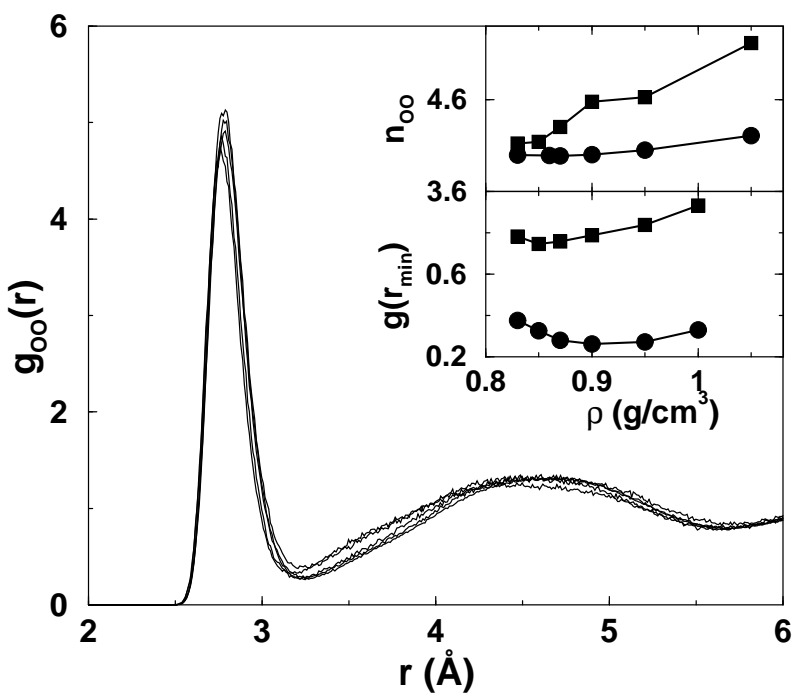

FIG. 2: Pair correlation function $g_{O O}(r)$ at $T=220 \mathrm{~K}$ and the same densities as Fig. 1. In the inset on the top number of nearest neighbours $n_{O O}$ as a function of $\rho$ for $T=300 \mathrm{~K}$ (full squares) and $T=220 \mathrm{~K}$ (full circles); in the inset below value of the first minimum of $g_{O O}(r), g\left(r_{\min }\right)$, as a function of $\rho$ at $T=300 \mathrm{~K}$ (full squares) and $T=220 \mathrm{~K}$ (full circles).

where $K_{T}$ is the isothermal compressibility defined as

$$
K_{T}=\frac{1}{\rho}\left(\frac{\partial \rho}{\partial P}\right)_{T}
$$

In the phenomenological description of the liquid-gas transition, as given for instance by mean field theories like the Van der Waals equation, along an isotherm the limits of the mechanical stability are marked by the changes in the slope of the $P_{T}(\rho)$ curve. Therefore from Eq. (3) it is found that the crossing to unstable states with $K_{T}<0$ takes place at singularity points where $K_{T}$ diverges. The spinodal line is identified by these singularity points. In particular the point where the minimum of $P_{T}(\rho)$ is located represents the limit of stability of the superheated liquid and so it belongs to the liquid branch of the spinodal.

We are interested here at the liquid spinodal which extends in the $P-T$ plane below the liquid-gas coexistence into the region of negative pressures. The liquid spinodal indicates also the limiting values of tension for the existence of an homogeneous fluid before phenomena like cavitation take place [2].

The behaviour of $P_{T}(\rho)$ for the BSV water is shown in Fig. 3 for $\rho$ ranging from 1.05 to $0.83 \mathrm{gr} / \mathrm{cm}^{3}$. In the inset of Fig. 3 four isotherms are represented upon lowering the temperature from $T=350 \mathrm{~K}$ to $T=260 \mathrm{~K}$. Isotherms from $T=240 \mathrm{~K}$ to $T=210 \mathrm{~K}$ are shown in the main frame of Fig. 3 From $T=300 \mathrm{~K}$ to the lowest temperature investigated the $P_{T}(\rho)$ curves exhibit a minimum in the simulated range of densities. The isotherms corresponding to lower temperatures have larger fluctuations due to the influence of both the low temperatures 
and the negative pressures In order to better follow the curves for the lowest temperatures shown in Fig. 31 we fitted the calculated points of the isotherms with a fourthorder polynomial function. We observe however that due to the fluctuations the fit systematically slightly overestimates the values of the minima. Therefore we will consider the minima directly observed for the plot of the spinodal line.

Based on Eq. 2 the minima of the isotherms represent the limit of stability of the superheated liquid and identify the values of the pressure at the spinodal, $P_{s}(T)$. As $T$ decreases $P_{s}(T)$ clearly shifts to larger values of $\rho$. This behaviour is similar to that observed for TIP5P 16] while for ST2 and TIP4P this shift is less evident 11].

The resulting estimated liquid spinodal line $P_{s}(T)$ is plotted in fig. 4. In the same figure also the $P_{\rho}(T)$ isochores are shown as obtained by the data set of tables [ and II. At least down to the lowest investigated temperature, $T=210 \mathrm{~K}$, the spinodal line is not retracing. In the SLC framework the spinodal line is expected to become reentrant at the point where it intersects the TMD line in the phase diagram. We recall that this behaviour is required if the TMD line has a negative slope [7, 11, 39].

Along the TMD line the coefficient of thermal expansion

$$
\alpha_{P}=\frac{1}{V}\left(\frac{\partial V}{\partial T}\right)_{P}
$$

goes to 0 . Since the thermal pressure coefficient $\gamma_{V}$

$$
\gamma_{V}=\left(\frac{\partial P}{\partial T}\right)_{V}
$$

is connected to the coefficient $\alpha_{P}$ by the following relation

$$
\gamma_{V}=\frac{\alpha_{P}}{K_{T}}
$$

the TMD points are on the line connecting the minima of the estimated $P_{\rho}(T)$ isochore for different $\rho$.

According to Fig. 4 as $\rho$ decreases, approaching the spinodal line, the isochores exhibit a minimum at smaller values of $T$.

The isochore for the lowest reported density $\rho=$ $0.90 \mathrm{gr} / \mathrm{cm}^{3}$ does not show any minimum in the range of $T$ investigated where instead the minima for the other isochores are found. The TMD line does not approach the spinodal line and at the lower $T$ investigated it results to have a positive slope. This behaviour of the TMD line prevents an intersection with the spinodal at negative $\mathrm{P}$ and low $\mathrm{T}$, unless a further change in the slope of the TMD line is hypothized.

These results are in agreement with the findings of the studies done on non polarizable models for water [11, 12, 13, 14, 15, 16. In fact for all these models the TMD line changes slope to avoid intersection with a non reentrant spinodal. Differences among the models can be found in the values of pressures and temperatures where the curves span. In particular the change of slope of the

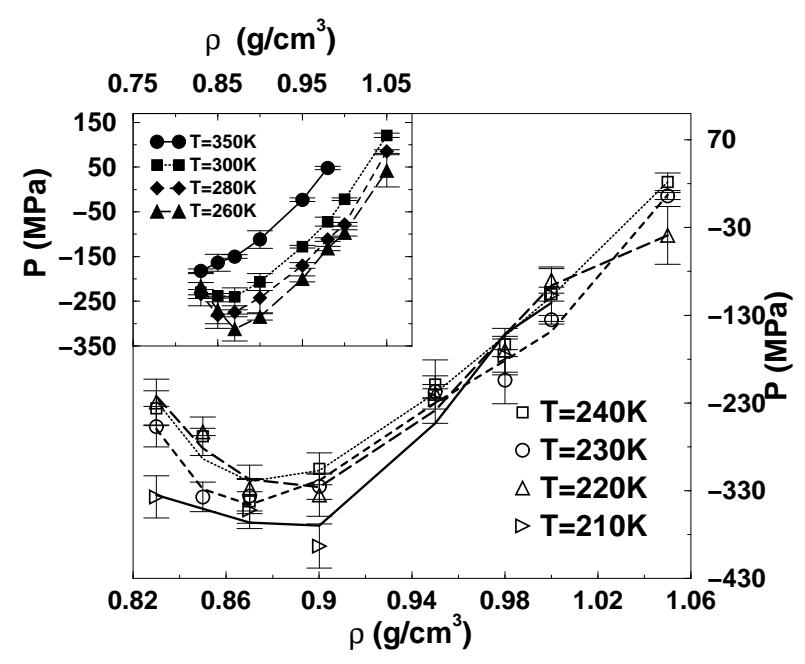

FIG. 3: Isotherms (symbols) for densities ranging from 1.05 to $0.83 \mathrm{gr} / \mathrm{cm}^{3}$. Errors are calculated with the method of statistical inefficiency [38]. In the inset temperatures range from $350 \mathrm{~K}$ to $260 \mathrm{~K}$. In the main frame temperatures range from $240 \mathrm{~K}$ to $210 \mathrm{~K}$ and lines are fit with a fourth order polynomial function.

TMD line appears in our model at negative pressures, $P<-120 \mathrm{MPa}$, similar to SPC/E 15], $P<-80 \mathrm{MPa}$ and to ST2 [11]. For TIP5P [16] the change of slope appears at ambient pressure instead.

The most studied alternative to the SLC for explaining the anomalies of water is the presence of a second critical point. The SCP hypothesis [8, 14] is based on the consideration that in the glassy state two distinct types of structure are observed [40], the low density amorphous (LDA) ice and the high density amorphous (HDA) ice. This polymorphism of water would be extended into the liquid phase and it would emerge as a coexistence line between two liquid phases culminating in a second critical point [41]. Computer simulation of waterlike lattice models show evidence in fact of two immiscible liquid forms of water [42, 43, 44, 45].

More recently several amorphous ice phases [46] have been experimentally observed and signatures have been found in computer simulation of the existence of several liquid-liquid coexistences in supercooled water [47]. Also Gibbs ensemble Monte Carlo (GEMC) studies on the BSV water have found two liquid-liquid coexistence curves besides the liquid-vapor equilibrium [30]. The liquid branch of the gas-liquid coexistence curve obtained with the GEMC is reported in Fig. 5, where we collect the relevant features of the behaviour of the BSV water in the $(T, \rho)$ thermodynamical plane. The spinodal line obtained in the present work in the supercooled region is compatible with the GEMC results. It comes out that the metastability region explored here goes very close to the boundaries of the region where new phases of the liquid water have been found. 


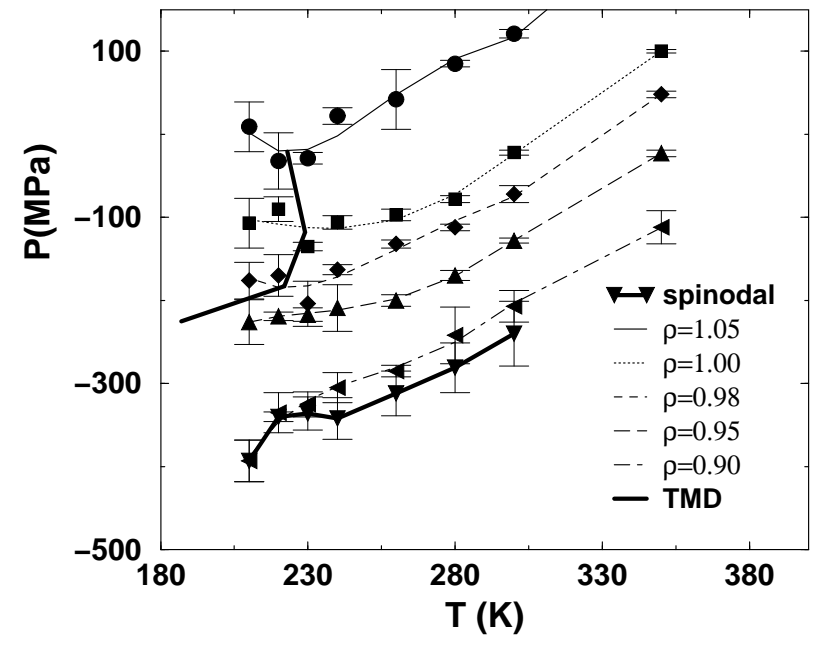

FIG. 4: $P_{\rho}(T)$ isochores for several values of $\rho$ in $\mathrm{g} / \mathrm{cm}^{3}$, spinodal line and TMD curve. As $\rho$ decreases, approaching the spinodal line, the isochores exhibit a minimum at smaller values of $T$. The spinodal line decreases upon lowering temperature and no retracing behaviour is observed.

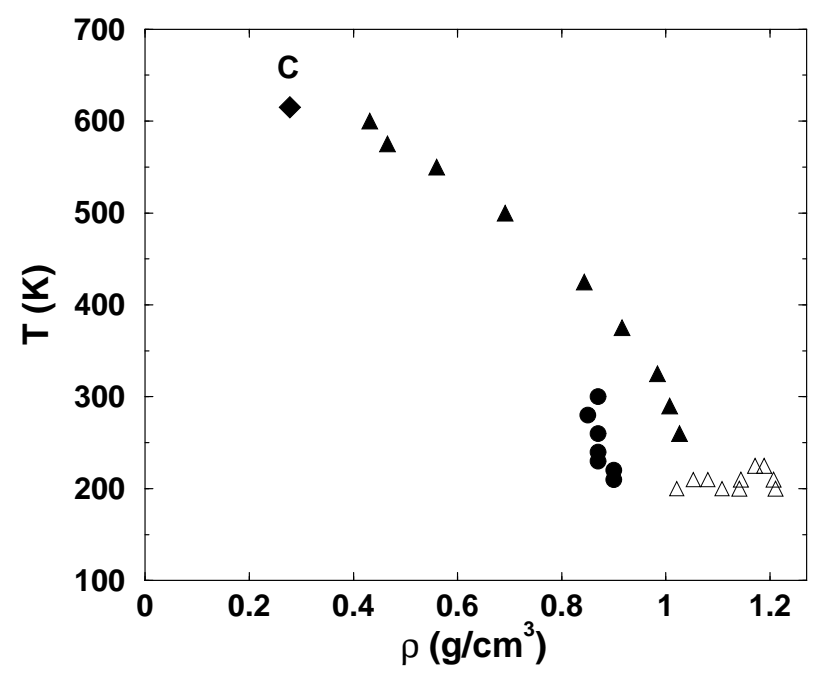

FIG. 5: Liquid branch of the coexistence curve obtained by GEMC [30] (filled triangles) and the spinodal line of the present work (black circles) in the $T, \rho$ plane for the BSV water. The diamond represents the estimated gas-liquid critical point $C$. Portions of the liquid-liquid coexistence regions are shown by the open triangles [30].

\section{CONCLUSIONS}

We have done Molecular Dynamics simulation of supercooled water described with a polarizable BSV poten- tial. The aim of the paper was to calculate the behaviour of the spinodal line and of the TMD line down to lowest possible temperatures and pressures.

We have performed a structural analysis of water described by the BSV polarizable potential in the region of the supercooled liquid and we have found that the system persists in an homogeneous phase down to $T=210 \mathrm{~K}$, $P \approx-390 \mathrm{MPa}$ and this occurs for $\rho \approx 0.9$. Signatures of the presence of the mechanical stability limit are observed in the behaviour of the values of the RDF at the first minimum.

The boundaries of the liquid mechanical stability at low $T$ and negative $P$ have been determined by the direct calculations of the liquid spinodal trough the minima of the isotherms $P_{T}(\rho)$. The line of the TMD in the same thermodynamical region has also been detected from the minima of the isochores $P_{\rho}(T)$.

We did not find any retracing of the spinodal line in the investigated region while we observe a change of slope of the TMD line which prevents for continuity a possible intersection of the TMD with the spinodal even down to lower $T$ and $P$. These results are in agreement with previous calculations done with non polarizable site potentials [11, 12, 13, 14, 15, 16]. Our analysis excludes indeed the possibility of the SLC scenario.

As a matter of fact the no retracing of the spinodal is compatible however with both the SI interpretation [10] and the possible existence of a second critical point [8]. On the other hand we have shown that the liquid spinodal calculated in the present work is in agreement with the gas-liquid coexistence curve obtained with the GEMC [30]. Since in the GEMC there are clear indications of the existence of two liquid-liquid coexistence regions we can infer that our results support the second critical point scenario.

The use of a polarizable potential in the present work has given an answer to two important issues. The first is that polarizable and non polarizable potentials are equally able to describe an unique, and therefore likely, scenario for the liquid-gas spinodal of supercooled water. This is an important achievement as the parameters of these potentials have been calculated through fit to experimental data performed at ambient conditions. Therefore the uniqueness of phase behaviour far from these conditions reinforces the possibility that these potentials are able to predict existing features of water. The second is that the analysis performed with the polarizable model can be extended in a different zone of the phase diagram for determining the liquid-liquid spinodal and indications about the second critical point location.
[1] C. A. Angell in Water: a comprehensive treatise, edited by F. Franks (Plenum Press, New York, 1981), vol. 7, p.
1; Annu. Rev. Phys. Chem. 34, 593 (1983).

[2] P.G. Debenedetti Metastable Liquids. Concepts and Prin- 
ciples Princeton, NJ, University Press. (1996).

[3] R. Speedy, P. G. Debenedetti, R. S. Smith, C. Huang, and B. D. Kay, J. Chem. Phys. 105, 240 (1996).

[4] R. S. Smith, B. D. Kay, Nature 398788 (1999).

[5] P.G. Debenedetti, J. Phys.: Condens. Matt. 15, R1670 (2003).

[6] R.J. Speedy, C.A. Angell, J. Phys. Chem. 65, 851 (1976).

[7] R. Speedy, J. Phys. Chem. 86982 (1982); ibid 86, 3002 (1982).

[8] P.H.Poole, F.Sciortino, U.Essmann and H.E.Stanley, Nature 360, 324 (1992).

[9] O. Mishima, H.E. Stanley Nature 392, 164 (1998); ibid. 396, 329 (1998).

[10] S. Sastry, P.G. Debenedetti, F. Sciortino and H. E. Stanley Phys. Rev. E 536144 (1996).

[11] P.H.Poole, F.Sciortino, U.Essmann and H.E.Stanley, Phys. Rev. E 48, 3799 (1993)

[12] F. Sciortino, P.H. Poole, U. Essmann and H. E. Stanley, Phys. Rev. E 55, 727 (1997).

[13] P. H. Poole, I. Saika-Voivod and F. Sciortino J. Phys. Condens. Matter 17, L431 (2005).

[14] H. Tanaka, J. Chem. Phys. 105, 5099 (1996).

[15] S. Harrington, P.H. Poole, F. Sciortino and H.E. Stanley, J. Chem. Phys. 107, 7443 (1997).

[16] M.Yamada, S. Mossa, H.E. Stanley, F.Sciortino, Phys. Rev. Lett. 88,195701 (2002)

[17] M.Sprik and M. L. Klein, J. Chem. Phys. 89, 7556 (1988).

[18] P. Ahlstrom, A. Wallqvist, S. Engstrom, B. Jonsson, Mol. Phys. 68, 563 (1989).

[19] D. van Belle, M. Froeyen, G. Lippens, and S. J. Wodak, Mol. Phys. 77, 239 (1992).

[20] R. E. Kozack and P. C. Jordan, J. Chem. Phys. 96, 3120 (1992).

[21] S. W. Rick, S. J. Stuart and B. J. Berne, J. Chem. Phys. 101, 6141 (1994).

[22] G. Ruocco and M. Sampoli, Mol. Phys. 82, 875 (1994).

[23] Brodholt J., Sampoli M., and Vallauri R., 1995, Mol. Phys., 86, 149 (1995).

[24] P. G. Kusalik, F. Liden, and I. M. Svishchev, J. Chem. Phys. 103, 10169 (1995).

[25] A. A. Chialvo and P. T. Cummings, J. Chem. Phys. 105, 8274 (1996).

[26] L. X. Dang and T. M. Chang, J. Chem. Phys. 106, 8149 (1997).

[27] K. Kiyohara, K. E. Gubbins and A. Z. Panagiotopolous, Mol. Phys. 94, 803 (1998).

[28] B. Guillot and Y. Guissani, J. Chem. Phys. 114, 6720 (2001).

[29] H. B. Yu, T. Hansson, W. F. van Gunsteren, J. Chem. Phys. 118, 221 (2003).

[30] P.Jedlovszky and R. Vallauri, J. Chem. Phys. 122, 081101 (2005).

[31] P.Jedlovszky, R.Vallauri, J. Chem. Phys. 115, 3750 (2001).

[32] P.Jedlovszky, R.Vallauri, Phys.Rev.E 67011201 (2003).

[33] Jedlovszky P., Brodholt J. P., Bruni F., Ricci M. A., Soper A. K., and Vallauri R., 1998, J. Chem. Phys., 108, 8528.

[34] P.Jedlovszky, R.Vallauri, Mol.Phys.97(1999) 1157

[35] P.Jedlovszky, J.Richardi, J.Phys.Chem. 110, 8019 (1999)

[36] G. Ruocco and M. Sampoli, Mol. Sim. 15, 281 (1995).

[37] A. K. Soper, J. Chem. Phys. 101, 6888 (1994).

[38] M. P. Allen and D. J. Tildesley, Computer Simulation of Liquids, Clarendon Press, Oxford (1987).
[39] M. C. D'Antonio, P. G. Debenedetti, J.Chem.Phys. 86, 2229 (1987).

[40] O. Mishima, J.Chem.Phys. 100, 5910 (1994).

[41] O.Mishima, Phys. Rev. Lett. 85, 334 (2000).

[42] S. Sastry, F. Sciortino, H.E. Stanley. J.Chem.Phys. 98, 9863 (1993).

[43] S.S. Borick, P. G. Debenedetti, S. Sastry, J. Phys. Chem. 99, 3781 (1995).

[44] C. J. Roberts, A. Z. Panagiotopoulos, P. G. Debenedetti, Phys. Rev. Lett. 77, 4386 (1996).

[45] C. J. Roberts, P. G. Debenedetti, J. Chem. Phys. 105, 658 (1996).

[46] C. A. Tulk, C. J. Benmore, J. Urquidi, D. D. Klug, J. Neuefeind, B. Tomberli and P. A. Egelstaff, Science 297, 1320 (2002).

[47] I. Brovchenko, A. Geiger, A. Oleinikova, J. Chem. Phys. 118, 9473 (2003). 


\begin{tabular}{|r|r|r|r|}
\hline \hline$T(K)$ & $\rho\left(g / c m^{3}\right)$ & $P \pm \delta P(M P a)$ & $U \pm \delta U(K J / m o l)$ \\
\hline \hline 350 & 1.00 & $100 \pm 2$ & $-39.16 \pm 0.17$ \\
350 & 0.98 & $48 \pm 24$ & $-39.03 \pm 0.18$ \\
350 & 0.95 & $-23 \pm 4$ & $-37.63 \pm 0.04$ \\
350 & $0.90^{*}$ & $-112 \pm 40$ & $-37.28 \pm 0.04$ \\
350 & 0.87 & $-150 \pm 4$ & $-36.34 \pm 0.18$ \\
350 & $0.86^{*}$ & $-159 \pm 4$ & $-36.65 \pm 0.06$ \\
350 & 0.85 & $-164 \pm 38$ & $-36.52 \pm 0.06$ \\
350 & 0.83 & $-182 \pm 4$ & $-35.58 \pm 0.04$ \\
\hline 300 & 1.05 & $121 \pm 5$ & $-42.92 \pm 0.13$ \\
300 & 1.00 & $-22 \pm 3$ & $-41.55 \pm 0.10$ \\
300 & 0.98 & $-72 \pm 10$ & $-42.11 \pm 0.05$ \\
300 & 0.95 & $-128 \pm 20$ & $-40.97 \pm 0.02$ \\
300 & $0.90^{*}$ & $-207 \pm 37$ & $-40.76 \pm 0.15$ \\
300 & 0.87 & $-240 \pm 37$ & $-39.72 \pm 0.04$ \\
300 & $0.86^{*}$ & $-243 \pm 25$ & $-40.02 \pm 0.07$ \\
300 & 0.85 & $-238 \pm 5$ & $-39.49 \pm 0.04$ \\
300 & 0.83 & $-230 \pm 6$ & $-39.02 \pm 0.16$ \\
\hline 280 & 1.05 & $85 \pm 4$ & $-44.10 \pm 0.14$ \\
280 & 1.00 & $-78 \pm 4$ & $-43.25 \pm 0.04$ \\
280 & 0.98 & $-112 \pm 4$ & $-43.77 \pm 0.08$ \\
280 & 0.95 & $-170 \pm 6$ & $-42.56 \pm 0.06$ \\
280 & $0.90^{*}$ & $-242 \pm 34$ & $-42.77 \pm 0.06$ \\
280 & 0.87 & $-274 \pm 5$ & $-41.62 \pm 0.09$ \\
280 & $0.86^{*}$ & $-280 \pm 4$ & $-41.71 \pm 0.10$ \\
280 & 0.85 & $-281 \pm 30$ & $-41.06 \pm 0.12$ \\
280 & 0.83 & $-234 \pm 26$ & $-41.57 \pm 0.11$ \\
\hline 260 & 1.05 & $42 \pm 36$ & $-45.90 \pm 0.10$ \\
260 & 1.00 & $-97 \pm 7$ & $-44.91 \pm 0.03$ \\
260 & 0.98 & $-132 \pm 5$ & $-45.67 \pm 0.11$ \\
260 & 0.95 & $-200 \pm 7$ & $-44.47 \pm 0.08$ \\
260 & $0.90^{*}$ & $-285 \pm 7$ & $-44.65 \pm 0.10$ \\
260 & 0.87 & $-312 \pm 27$ & $-44.26 \pm 0.08$ \\
260 & $0.86^{*}$ & $-300 \pm 21$ & $-44.18 \pm 0.09$ \\
260 & 0.85 & $-270 \pm 30$ & $-44.38 \pm 0.06$ \\
260 & 0.83 & $-216 \pm 28$ & $-43.90 \pm 0.08$ \\
\hline \hline
\end{tabular}

TABLE I: Temperature, density, pressure and internal energy of the simulated state points. 


\begin{tabular}{|c|c|c|c|}
\hline$T(K)$ & $\rho\left(\mathrm{g} / \mathrm{cm}^{3}\right)$ & $P \pm \delta P(M P a)$ & $U \pm \delta U(K J / \mathrm{mol})$ \\
\hline 240 & 1.05 & $22 \pm 10$ & $-47.09 \pm 0.08$ \\
\hline 240 & 1.00 & $-106 \pm 8$ & $-46.81 \pm 0.06$ \\
\hline 240 & 0.98 & $-163 \pm 6$ & $-47.48 \pm 0.09$ \\
\hline 240 & 0.95 & $-209 \pm 28$ & $-46.52 \pm 0.06$ \\
\hline 240 & $0.90^{*}$ & $-305 \pm 18$ & $-46.87 \pm 0.14$ \\
\hline 240 & 0.87 & $-342 \pm 25$ & $-45.82 \pm 0.08$ \\
\hline 240 & $0.86^{*}$ & $-329 \pm 18$ & $-46.23 \pm 0.06$ \\
\hline 240 & 0.85 & $-268 \pm 22$ & $-45.78 \pm 0.07$ \\
\hline 240 & 0.83 & $-236 \pm 20$ & $-46.42 \pm 0.08$ \\
\hline 230 & 1.05 & $6 \pm 4$ & $-47.95 \pm 0.03$ \\
\hline 230 & 1.00 & $-135 \pm 5$ & $-48.26 \pm 0.03$ \\
\hline 230 & 0.98 & $-204 \pm 27$ & $-47.87 \pm 0.09$ \\
\hline 230 & 0.95 & $-217 \pm 8$ & $-47.62 \pm 0.09$ \\
\hline 230 & $0.90^{*}$ & $-325 \pm 24$ & $-47.26 \pm 0.09$ \\
\hline 230 & 0.87 & $-336 \pm 20$ & $-46.67 \pm 0.07$ \\
\hline 230 & $0.86^{*}$ & $-330 \pm 10$ & $-47.52 \pm 0.10$ \\
\hline 230 & 0.85 & $-337 \pm 17$ & $-46.63 \pm 0.08$ \\
\hline 230 & 0.83 & $-257 \pm 23$ & $-46.14 \pm 0.09$ \\
\hline 220 & 1.05 & $-39 \pm 33$ & $-49.67 \pm 0.08$ \\
\hline 220 & 1.00 & $-90 \pm 15$ & $-48.74 \pm 0.08$ \\
\hline 220 & 0.98 & $-170 \pm 25$ & $-48.87 \pm 0.09$ \\
\hline 220 & 0.95 & $-219 \pm 5$ & $-48.18 \pm 0.05$ \\
\hline 220 & $0.90^{*}$ & $-335 \pm 24$ & $-48.29 \pm 0.08$ \\
\hline 220 & 0.87 & $-327 \pm 26$ & $-47.95 \pm 0.05$ \\
\hline 220 & $0.86^{*}$ & $-339 \pm 14$ & $-47.78 \pm 0.08$ \\
\hline 220 & 0.85 & $-305 \pm 11$ & $-46.85 \pm 0.07$ \\
\hline 220 & 0.83 & $-229 \pm 26$ & $-47.38 \pm 0.05$ \\
\hline 210 & 1.05 & $9 \pm 30$ & $-50.09 \pm 0.09$ \\
\hline 210 & 1.00 & $-107 \pm 30$ & $-49.71 \pm 0.07$ \\
\hline 210 & 0.98 & $-176 \pm 22$ & $-49.53 \pm 0.08$ \\
\hline 210 & 0.95 & $-226 \pm 27$ & $-49.150 \pm 0.08$ \\
\hline 210 & $0.90^{*}$ & $-393 \pm 25$ & $-48.88 \pm 0.15$ \\
\hline 210 & 0.87 & $-352 \pm 21$ & $-48.39 \pm 0.09$ \\
\hline 210 & $0.86^{*}$ & $-253 \pm 30$ & $-49.10 \pm 0.11$ \\
\hline 210 & 0.85 & $-263 \pm 4$ & $-47.56 \pm 0.08$ \\
\hline 210 & 0.83 & $-337 \pm 24$ & $-47.86 \pm 0.08$ \\
\hline
\end{tabular}

TABLE II: Temperature, density, pressure and internal energy of the simulated state points. 